\title{
Łukasz Iluk
}

Wyższa Szkoła Finansów i Prawa in Bielsko-Biała, Bielsko-Biała

DOI: $10.19195 / 0435-5865.141 .15$

\section{Zur Frage der Adäquatheit der Übersetzungs- vorschläge in bilingualen Rechtswörterbüchern aus juristischer Sicht}

\section{Einleitung}

Das Übersetzen juristischer Texte oder die Entwicklung zweisprachiger Rechtswörterbücher verlangt u.a. gute Kenntnisse der Rechtsterminologie und ihrer Bedeutungsumfänge, die in den Ausgangs- und Zielrechtsordnungen gelten. Da Übersetzer in der Regel keine Fachleute auf dem behandelten Gebiet sind, müssen sie entsprechende terminologische Wörterbücher zu Rate ziehen, zumal sie eine begrenzte Möglichkeit zu einer eingehenden terminologischen Recherche haben. Aus diesem Grunde sind zweisprachige terminologische Wörterbücher eine wichtige, nicht selten die einzige terminologische Informationsquelle für Übersetzer. Diese Feststellung bestätigen u.a. die empirischen Untersuchungen von Małgorzewicz (2012: 183 ff.) zur Wörterbuchbenutzung angehender und praktizierender Übersetzer oder die von Nord (2002: 175).

Im folgenden Beitrag wollen wir am Beispiel eines Begriffsfeldes juristische Aspekte darstellen, die für eine übersetzungsorientierte terminologische Analyse von besonderer Relevanz sind. Daraus ergeben sich methodologische Hinweise für terminologische und lexikographische Analysen.

\section{Qualität bilingualer Rechtswörterbücher}

Der Übersetzer greift nach bilingualen Fachwörterbüchern in der Regel mit der subjektiven Überzeugung, dass die darin angegebenen bilingualen Wortgleichungen verifiziert und somit zuverlässig sind. Aus der von de Groot und van Laer 
(2008: 1) durchgeführten Analyse von mehr als 170 zweisprachigen Rechtswörterbüchern geht jedoch hervor, dass ihre Qualität dürftig bis extrem schlecht ist.

[...] publishing houses are offering numerous bilingual legal dictionaries to translators and lawyers. To translate between the different languages of the Member States of the European Union (EU) about one hundred seventy bilingual legal dictionaries are available. Regrettably, the quality of most of these dictionaries is poor to extremely bad (de Groot \& van Laer 2008: 1).

Aus ihrer groß angelegten Untersuchung ergibt sich die dringende Notwendigkeit, die Angaben zu terminologischen Äquivalenzrelationen in vorhandenen Rechtswörterbüchern zu verifizieren, zu ergänzen, notfalls zu korrigieren, zumal die in Fachwörterbüchern formulierten Übersetzungsvorschläge eine standardisierende (normierende) Funktion ausüben (de Groot 1999: 42). Wie weiter gezeigt wird, sind das keine Einzelfälle.

\section{Methodisches Vorgehen bei konfrontativen Begriffsanalysen für übersetzerische und lexikographische Zwecke aus juristischer Sicht}

Die korrekte Verwendung juristischer Termini (Benennungen) setzt fundiertes Fachwissen voraus. Dazu gehört u.a. das Begriffsfeldwissen, das Begriffe aus einem Sachfach, ihre Begriffsintension und -extension umfasst. Es erfasst auch die systematischen Beziehungen, in denen die Begriffe eines Sachgebiets stehen (Morgenroth 1987: 115). Die Kenntnis der Begriffsfelder ist umso wichtiger, als eine absolute begriffliche Äquivalenz zwischen unterschiedlichen Rechtssystemen nicht vorhanden ist. Um eine zumindest approximative Äquivalenz im Sandrinis (1996: 145) Sinne zu ermitteln, ist eine vorausgehende, übersetzerorientierte, komparative Begriffsanalyse notwendig. Im Weiteren wollen wir zeigen, welche Aspekte dabei zu berücksichtigen sind und wie methodisch vorzugehen ist.

\subsection{Auswahl eines repräsentativen Textkorpus}

Da der Gebrauch juristischer Termini durch präskriptive Normen geregelt ist, dürfen keine beliebigen Fachtexte für deren Ermittlung ausgewertet werden, sondern nur solche, die den Status haben, die Gebrauchsnormen für Rechtstermini zu setzen. Dieser Status kommt Gesetzestexten und eventuell Urteilen der höchsten Gerichte zu, da sie präskriptiv die Bedeutung, die Form und den Gebrauch juristischer Benennungen bestimmen. Wichtig ist es dabei, alle Sparten des Rechts zu 
berücksichtigen, da dieselben Benennungen in unterschiedlichen Rechtsgebieten mit anderen Begriffsinhalten oder unterschiedliche Benennungen mit ähnlichen Begriffsinhalten verwendet werden.

\subsection{Festlegung eines zu untersuchenden Begriffsfeldes}

In jedem Rechtssystem herrschen komplizierte begriffliche Strukturen, da dieselben Benennungen in unterschiedlichen Rechtsgebieten mit mehr oder wenigen unterschiedlichen Inhalten gebraucht werden. Deshalb ist es hier kaum möglich, einwandfreie hierarchische Begriffsysteme zu erstellen, wie das etwa in naturwissenschaftlichen Fachgebieten der Fall ist. Aus diesem Grunde eignet sich hierfür die Erstellung von Begriffsfeldern (Arntz/Picht 1991: 112). Nach der DIN-Norm 2343 ist ein Begriffsfeld die "Menge von Begriffen, die zueinander in Beziehung stehen". Bei der Untersuchung eines Begriffsfeldes geht man von einem Oberbegriff aus und fragt nach den dazugehörigen Benennungen. Ein Begriffsfeld konstituieren somit Termini, die thematische Nähe, aber oft eine relativ lockere Struktur aufweisen. Analysen von Begriffsfeldern haben einen punktuellen Charakter und helfen Begriffe in eine übersichtliche Ordnung zu bringen, sie semantisch voneinander abzugrenzen sowie komparativ zu analysieren. Solche Untersuchungen haben nach Arntz, Picht und Mayer (2004: 227) einen deskriptiven Charakter, da sie den bestehenden Fachwortbestand beschreibend zu erfassen versuchen.

\subsection{Feststellung terminologischer Äquivalenz von Rechtsbegriffen}

Ein grundlegendes Problem in komparativen terminologischen Untersuchungen ist die begriffliche Äquivalenz. Sie liegt nach Nordterm 13 vor, wenn zwei Benennungen aus zwei verschiedenen Sprachen denselben oder einen ähnlichen Begriff bezeichnen (Dobrina online: 25). Diese Feststellung weist darauf hin, dass Begriffe von einer Sprache unabhängig sind. Eine so verstandene begriffliche Äquivalenz gilt jedoch nicht für Rechtssprachen, da Rechtsbegriffe jeweils im Rahmen einer gegebenen Rechtsordnung in Übereinstimmung mit ihren Regelungsrichtlinien und Rechtsprinzipien durch Legaldefinitionen festgesetzt werden. Aus diesem Grunde kann man davon ausgehen, dass eine vollständige begriffliche Übereinstimmung von nationalen Rechtsbegriffen kaum gegeben ist. Um den Äquivalenzgrad zwischen Rechtsbegriffen aus zwei Rechtsordnungen festzustellen, werden in erster Linie ihre Begriffsmerkmale miteinander verglichen und bewertet. Nach Stolze (1999: 49) kann man Rechtsbegriffe aus zwei verschiedenen Rechtsordnungen für Äquivalent halten, wenn ihr Begriffsinhalt ein gemeinsames Minimum aufweist. Dieses Kriterium erfüllen die im Weiteren untersuchten deutschen und polnischen Benennungen: Jugendlicher, Minderjähriger, Heranwachsender, nieletni, mtodo- 
ciany, matoletni. Ihr gemeinsames begriffliches Minimum besteht darin, dass sie eine Person bezeichnen, die nicht volljährig ist. Sandrini (1996: 140-145) vertritt dagegen in seiner Monographie „Terminologiearbeit im Recht” die berechtigte Meinung, dass die höchste Äquivalenzstufe dann gegeben ist, wenn die Intensionen von Rechtsbegriffen aus zwei verschiedenen Rechtsordnungen deckungsgleich sind. Ihre Extensionen brauchen dagegen nicht deckungsgleich zu sein, weil sie von Rechtsprechungen im jeweiligen Land unterschiedlich festgesetzt werden können. Aus diesem Grunde setzt Übersetzen juristischer Fachtexte immer einen Rechtsvergleich und Sprachvergleich voraus (Simonnœs 1999: 140). Der Rechtsvergleich im Rahmen eines Begriffsfeldes ermöglicht, wie weiter unten gezeigt wird, das treffende Äquivalent in der Zielrechtsordnung präzise zu ermitteln.

\subsection{Beispiel einer Begriffsanalyse mit Hilfe eines Begriffsfeldes}

Unser Vorhaben wollen wir am Beispiel des Begriffsfeldes: Kind, Minderjähriger, Jugendlicher und Heranwachsender und ihrer polnischen Äquivalente illustrieren. Die das Begriffsfeld konstituierenden Termini stammen aus unterschiedlichen Rechtsgebieten, in denen ihre begrifflichen Inhalte durch Legaldefinitionen festgesetzt werden. Einer komparativen Untersuchung muss eine deskriptive Analyse ihrer begrifflichen Merkmale in der jeweiligen Rechtssprache vorausgehen.

\subsection{Juristische Deskription der untersuchten Begriffe}

Bei der Begriffsdeskription ist sowohl auf den Begriffsinhalt als auch auf das Rechtsgebiet, in dessen Rahmen der jeweilige Begriff definiert wurde, zu achten.

Kontextbelege mit Legaldefinitionen im deutschen Recht:

Der Terminus Jugendlicher begegnet uns im Strafrecht und Arbeitsrecht. Im Strafgesetzbuch wird er wie folgt definiert:

$\S 1$ des Jugendgerichtsgesetzes: Persönlicher und sachlicher Anwendungsbereich

(1) Dieses Gesetz gilt, wenn ein Jugendlicher oder ein Heranwachsender eine Verfehlung begeht, die nach den allgemeinen Vorschriften mit Strafe bedroht ist.

(2) Jugendlicher ist, wer zur Zeit der Tat vierzehn, aber noch nicht achtzehn, Heranwachsender, wer zur Zeit der Tat achtzehn, aber noch nicht einundzwanzig Jahre alt ist. ${ }^{1}$

$\S 10$ des Strafgesetzbuches

${ }^{1}$ Jugendgerichtsgesetz in der Fassung der Bekanntmachung vom 11. Dezember 1974 (BGB1. I S. 3427), das zuletzt durch Artikel 3 des Gesetzes vom 26. Juni 2013 (BGBl. I S. 1805) geändert worden ist. 
Sondervorschriften für Jugendliche und Heranwachsende für Taten von Jugendlichen und Heranwachsenden gilt dieses Gesetz nur, soweit im Jugendgerichtsgesetz nichts anderes bestimmt ist. ${ }^{2}$

Im Gesetz zum Schutz der arbeitenden Jugend wird Jugendlicher wie folgt definiert:

$\S 2$ des Jugendarbeitsschutzgesetzes: Kind, Jugendlicher

(1) Kind im Sinne dieses Gesetzes ist, wer noch nicht 15 Jahre alt ist.

(2) Jugendlicher im Sinne dieses Gesetzes ist, wer 15, aber noch nicht 18 Jahre alt ist.

(3) Auf Jugendliche, die der Vollzeitschulpflicht unterliegen, finden die für Kinder geltenden Vorschriften Anwendung. ${ }^{3}$

$\S 106$ des Bürgerlichen Gesetzbuches: Beschränkte Geschäftsfähigkeit Minderjähriger:

Ein Minderjähriger, der das siebente Lebensjahr vollendet hat, ist nach Maßgabe der $\S \S 107$ bis 113 in der Geschäftsfähigkeit beschränkt.

Kontextbelege mit Legaldefinitionen im polnischen Recht:

Art. $115 \S 10$ des polnischen Strafgesetzbuchs (Kodeks karny)

Młodocianym jest sprawca, który w chwili popełnienia czynu zabronionego nie ukończył 21 lat i w czasie orzekania w pierwszej instancji 24 lat.

Art. 10

$\S 1$. Na zasadach określonych w tym kodeksie odpowiada ten, kto popełnia czyn zabroniony po ukończeniu 17 lat.

$\S 2$. Nieletni, który po ukończeniu 15 lat dopuszcza się czynu zabronionego określonego w art. 134, art. $148 \S 1,2$ lub 3, art. $156 \S 1$ lub 3, art. $163 \S 1$ lub 3, art. 166, art. $173 \S 1$ lub 3, art. $197 \S 3$ lub 4, art. $223 \S 2$, art. $252 \S 1$ lub 2 oraz w art. 280, może odpowiadać na zasadach określonych w tym kodeksie, jeżeli okoliczności sprawy oraz stopień rozwoju sprawcy, jego właściwości i warunki osobiste za tym przemawiają, a w szczególności, jeżeli poprzednio stosowane środki wychowawcze lub poprawcze okazały się bezskuteczne.

Art. 200

$\S 1$. Kto obcuje płciowo z małoletnim poniżej lat 15 lub dopuszcza się wobec takiej osoby innej czynności seksualnej lub doprowadza ją do poddania się takim czynnościom albo do ich wykonania [...].

Polnisches Familien- und Vormundschaftsgesetzbuch (Kodeks rodzinny i opiekuńczy)

2 Strafgesetzbuch in der Fassung der Bekanntmachung vom 13. November 1998 (BGBl. I S. 3322), das zuletzt durch Artikel 1 des Gesetzes vom 23. April 2014 (BGB1. I S. 410) geändert worden ist.

${ }^{3}$ Gesetz zum Schutz der arbeitenden Jugend (Jugendarbeitsschutzgesetz) vom 12. April 1976 (BGB1. I S. 965), das zuletzt durch Artikel 3 Absatz 7 des Gesetzes vom 20. April 2013 (BGB1. I S. 868) geändert worden ist. 
Art. 170 Gdy małoletni osiągnie pełnoletność, albo gdy przywrócona zostanie nad nim władza rodzicielska, opieka ustaje z mocy prawa.

Polnisches Arbeitsgesetzbuch (Kodeks pracy)

Art. 190.

$\S 1$. Młodocianym w rozumieniu kodeksu jest osoba, która ukończyła 16 lat, a nie przekroczyła 18 lat.

Aus der Gegenüberstellung der Legaldefinitionen ergeben sich klare Übereinstimmungen und Differenzen. Die letzteren betreffen die Begriffsextensionen. Sandrinis Meinung folgend, spielen sie bei der Ermittlung des Äquivalenzgrades eine untergeordnete Rolle, solange sie im der Zielrechtsordnung nicht grundlegend anders kodifiziert wurden. Aus diesem Grunde können wir annehmen, dass die untersuchten Benennungen auf der intensionalen Ebene weitgehend äquivalent sind. Eine besondere Anmerkung gilt dem Begriff Heranwachsender und mtodociany. Gemäß den Legaldefinitionen handelt es sich um eine bereits volljährige Person, die im deutschen und polnischen Strafrecht aus erziehungspolitischen Gründen wie ein jugendlicher Straftäter behandelt werden kann.

\subsection{Distribution der Rechtstermini}

Die grundlegenden Unterschiede zwischen den analysierten Termini liegen in ihrer Distribution. Aus diesem Grunde darf sie in komparativen Untersuchungen nicht aus dem Blickfeld geraten. Unter Distribution verstehen wir alle Umgebungen, hier Rechtsgebiete, in denen ein Rechtsterminus gebraucht wird. Wie es sich im Weiteren zeigen wird, liefert die Distributionsanalyse der Rechtstermini wichtige Hinweise für die korrekte Verwendung eines Terminus. Die analysierten deutschen Begriffe kommen in folgenden Rechtsgebieten vor:

1) Jugendlicher im Strafrecht und Arbeitsrecht,

2) Minderjähriger (auch Kind, bzw. Kinder) im Zivilrecht und Familienrecht,

3) Heranwachsender nur im Strafrecht.

Ihre polnischen Äquivalente begegnen uns in folgenden Rechtsgebieten:

1) nieletni (im Sinne Jugendlicher) nur im Strafrecht,

2) matoletni (im Sinne Minderjähriger) im Strafrecht, Zivilrecht und Familienrecht,

3) mlodociany im Strafrecht (im Sinne Heranwachsender) und Arbeitsrecht (im Sinne Jugendlicher).

Aus der Gegenüberstellung ergeben sich klare Unterschiede in ihrer Distribution, die von den präskriptiven Normen der jeweiligen Rechtsgebiete festgesetzt wird. Diese Differenzen, solange sie dem Übersetzer unbekannt sind, verursachen gravierende Übersetzungsfehler. Ein Distributionsfehler einer Rechtsbenennung 
im Translat liegt dann vor, wenn bei deren Übersetzung entweder ein Nichtterminus oder ein Fachwort aus einem anderen Rechtsgebiet als in der Ausgangszielordnung verwendet wird. Als Beispiel bedienen wir uns der terminologischen Wortgleichung mlodociany - Heranwachsender im Sinne des Arbeitsrechts, wie sie im polnisch-deutschen Rechtswörterbuch von Kilian (2000: 165) angegeben wird. Dieser Übersetzungsvorschlag ist grundlegend falsch, weil er einen Terminus aus dem deutschen Strafrecht und nicht aus dem Arbeitsrecht enthält. Im Sinne des Arbeitsrechts ist nur die Wortgleichung mtodociany - Jugendlicher und in strafrechtlichen Texten die Wortgleichung mtodociany - Heranwachsender korrekt.

Ein anderes Beispiel: Das deutsche Jugendamt wird in Kilians deutsch-polnischen Rechtswörterbuch (1996: 322) mit urząd do spraw młodzieży übersetzt. Diese Äquivalenzangabe kann man aus zwei Gründen nicht akzeptieren:

1. Aus rechtlicher Sicht fungiert die polnische Bezeichnung młodzież (Jugend) nicht als juristischer Begriff.

2. Die Aufgaben des deutschen Jugendamtes werden durch das Familienrecht bestimmt und beziehen sich auf Schutz und Aufsicht von Minderjährigen (Abschnitt 3 des Bürgerlichen Gesetzbuches). ${ }^{4}$

Daher ist die Verwendung der Benennung młodzież in Kilians Übersetzungsvorschlag insofern inadäquat, als sie keinen Status eines Rechtsterminus hat und die Minderjährigkeit in der oberflächlichen Benennungsstruktur nicht explizit zum Ausdruck bringt. In Übereinstimmung mit den präskriptiven Normen des Familienrechts sollte Jugendamt im Polnischen mit urzad do spraw matoletnich (wortwörtlich Amt für Angelegenheiten der Minderjährigen) wiedergegeben werden. Die Benennung nieletni scheidet hier als Äquivalent aus, weil sie nur im Strafrecht vorkommt, während Jugendamt eine Institution des Familienrechts ist. Demzufolge verlangt eine Übersetzung eines Terminus oder einer seiner Konstituenten die Beachtung ihrer spezifischen Distribution in dem jeweiligen Rechtsgebiet der Ausgangs- und Zielrechtsordnung. Wie wir sehen, ist die Distributionskongruenz der Termini ein wichtiges Kriterium für die Ermittlung der Übersetzungsadäquatheit, da sie die materielle Äquivalenz der Benennungen aus zwei verschiedenen Rechtsordnungen sicherstellt. Distributionell inkongruente Übersetzungsvorschläge verstoßen gegen die präskriptiven Normen der jeweiligen Rechtsnormung. Aus diesem Grunde sollte jedes bilinguale Rechtswörterbuch bei einem Lemma die Rechtssparten eines Terminus und seines Äquivalents systematisch und nicht nur inzidentell angeben, um möglichen Distributionsfehlern vorzubeugen.

${ }^{4}$ Abschnitt 3 des Bürgerlichen Gesetzbuches in der Fassung der Bekanntmachung vom 2. Januar 2002 (BGB1. I S. 42, 2909; 2003 I S. 738), das zuletzt durch Artikel 4 Absatz 5 des Gesetzes vom 1. Oktober 2013 (BGBl. I S. 3719) geändert worden ist. 


\section{Konzeptualisierung von Rechtsbegriffen}

Einen wichtigen, aber oft übersehenen Aspekt in bilingualen terminologischen Rechtsvergleichen stellt das Problem der Begriffskonzeptualisierung dar. Die Termini małoletni und Minderjähriger bezeichnen im deutschen und polnischen Strafrecht das Opfer einer Straftat. Der nicht volljährige Straftäter wird dagegen im deutschen Strafrecht Heranwachsender oder Jugendlicher und im polnischen mtodociany oder nieletni genannt. Diesen Konzeptualisierungsunterschieden wird bei der Übersetzung semantisch motivierter Benennungen keine hinreichende Aufmerksamkeit geschenkt, was folglich inkorrekte Äquivalenzangaben in bilingualen Rechtswörterbüchern zu Folge hat. So zum Beispiel wird Jugendgefängnis im deutsch-polnischen Rechtswörterbuch von Kilian (1996: 323) irrtümlicherweise mit zakład karny dla nieletnich (wortwörtlich Gefängnis für Minderjährige) wiedergegeben, während das adäquate Äquivalent in Übereinstimmung mit der Begriffskonzeptualisierung in der polnischen Legaldefinition und terminologischen Distribution zakład karny dla młodocianych (wortwörtlich Gefängnis für Jugendliche) heißen soll (art. 84 KWK). ${ }^{5}$ Dieser Unterschied in der Benennung einer Strafanstalt im Polnischen ist die Folge der begrifflichen Differenzierung junger Straftäter, die sich aus den bildungspolitischen und erzieherischen Zielsetzungen des Strafvollzugs ergibt. Diesen Benennungsunterschied gibt es in der deutschen Rechtsordnung nicht.

\section{Schlussfolgerungen}

Die analysierten Beispiele von Übersetzungsvorschlägen aus einem bilingualen Rechtswörterbuch zeigen, dass zweisprachige Wortgleichungen keine korrekte terminologische Äquivalenz garantieren, wenn sie nur auf deren bedeutungsindizierenden Benennungsmotivik aufbauen. In diesem Zusammenhang warnt de Groot (1999: 18) davor, ,aus der Ausgangssprache in die umgangsprachliche Terminologie der Zielsprache“ zu übersetzen. Es soll ,grundsätzlich in die juristische Terminologie der Zielsprache übersetzt werden“. Eine ähnliche Meinung vertritt Horn-Helf (1999: 116-118). Nach ihrer Auffassung besteht die Herstellung einer Benennungsäquivalenz nicht im „Übersetzen”, sondern im „Ersetzen” ausgangssprachlicher durch zielsprachliche Termini. Die Inkorrektheit der weiter oben diskutierten Übersetzungsvorschläge besteht eben darin, dass sie keine Termini der Zielsprachenrechtsordnung, sondern umgangssprachliche Benennungen enthalten.

Im vorliegenden Beitrag wurde eine Methode präsentiert, nach der man Termini aus unterschiedlichen Rechtsordnungen für bilinguale lexikographische und

${ }^{5}$ Ustawa z dnia 20 maja 1971 r. Kodeks wykroczeń (Dz.U. 1971 Nr 12 poz. 114 z późn. zm.). 
übersetzerische Zielsetzungen zuverlässig ermitteln kann. In dieser Methode sind folgende Aspekte zu berücksichtigen:

- Auswahl eines repräsentativen Textkorpus,

- Ermittlung der Distribution der Rechtsbegriffe in unterschiedlichen Rechtssparten,

- Ermittlung einer bilingualen terminologischen Äquivalenz auf der Grundlage von Legaldefinitionen in Gesetzestexten und nicht allein auf der Grundlage von bedeutungsindizierender Benennungsmotivik,

- Abgleich der Konzeptualisierungen der Begriffsinhalte insbesondere in Bezug auf ihre teleologischen Merkmale,

- Bewertung des Grades der Begriffsidentität,

- Entscheidung für die jeweilige Übersetzungsstrategie bzw. Auswahl einer Benennung der Zielrechtsordnung.

Welcher Begriff und welche Benennungen im jeweiligen Rechtsgebiet und mit welcher Bedeutung verwendet werden, entscheiden die präskriptiven Normen des Gesetzgebers. Ihre strikte Berücksichtigung garantiert eine adäquatere Ersetzung von Termini einer Ausgangsrechtsordnung durch Termini der Zielrechtsordnung. Wie weiter oben gezeigt, hat der Übersetzer, Terminologe oder Lexikograph bei der Auswahl eines Begriffs aus einem Begriffsfeld den präskriptiven Normen der jeweiligen Rechtsordnung stets Rechnung zu tragen. ${ }^{6}$ Dasselbe gilt für juristische Fachwörterbücher, die den Benutzer nicht nur über die terminologische Äquivalenz, sondern auch über die jeweilige Sparte informieren sollten, in der die betroffene Benennung normgerecht oder usuell verwendet wird.

\section{Literatur}

Arntz, Reiner \& Picht, Herbert: Einführung in die Terminologiearbeit. 2. Auflage, Hildesheim 1991. Arnzt, Reiner \& Picht, Heribert \& Mayer, Felix: Einführung in die Terminologiearbeit. Hildesheim 2004.

De Groot, Gerard-René: Das Übersetzen juristischer Terminologie. In: G.-R. De Groot \& R. Schultze: Recht und Übersetzen. Baden-Baden 1999, S. 11-46.

De Groot, Gerard-René \& van Laer, Conrad (2008): The Quality of Legal Dictionaries: an assessment. In: Maastricht Working Paper. Faculty of Law. [online Zugriff am 20.06.2013].

Dobrina, Claudia: Die Suche nach der Äquivalenz: Auf einem Streifzug durch drei Disziplinen (online 20.06.2014).

Horn-Helf, Brigitte: Technisches Übersetzen in Theorie und Praxis. München 1999.

Jermol, Ada Gruntar: Übersetzen von Rechtstexten - einige didaktische Hinweise. In: Lebende Sprachen 2012, 57/1, s. 53-73.

Kilian, Alina: Stownik języka prawniczego i ekonomicznego niemiecko-polski. Warszawa 1996.

Kilian, Alina: Stownik języka prawniczego i ekonomicznego, polsko-niemiecki. Warszawa 2000.

${ }^{6}$ Auf andere Aspekte, wie etwa sprachliche Form der Benennungsäquivalente, kann hier aus Rahmengründen nicht näher eingegangen werden. 
Małgorzewicz, Anna: Die Kompetenzen des Translators aus kognitiver und translationsdidaktischer Sicht. Wrocław 2012.

Morgenroth, Klaus: Die Vermittlung betriebswirtschaftlicher Fachterminologie. In: Fremdsprachen lehren und lernen. Bd. 16. 1987, S. 111-132.

Nord, Christiane: Hilfsmittel beim Übersetzen: eine empirische Studie zum Rechercheverhalten professioneller Übersetzer. Frankfurt am Main 2002.

Sandrini Peter: Terminologiearbeit im Recht / Deskriptiver begriffsorientierter Ansatz vom Standpunkt des Übersetzers. Wien 1996.

Simonnœs, Ingrid: Zur Frage der rechtskulturellen Unübersetzbarkeit anhand eines Vergleichs zwischen Norwegen und Deutschland. In: Lars Eriksen / Karin Luttermann (Hrsg.) Juristische Fachsprache. Kongressberichte des 12th European Symposium on Language for Special Purposes. Brixen 1999, S. 139-156.

Stolze Radegundis: Expertenwissen des juristischen Fachübersetzers. In: Peter Sandrini (Hrsg.), Übersetzen von Rechtstexten - Fachkommunikation im Spannungsfeld zwischen Rechtsordnung und Sprache. Tübingen 1999, S. 45-62.

\section{Abstracts}

Im vorliegenden Artikel werden Probleme der Adäquatheit der Übersetzungsvorschläge in bilingualen Rechtswörterbüchern erörtert. Mit Hilfe von Legaldefinitionen und Begriffsfeldern werden die intensionale und distributionelle Kongruenz von Rechtstermini verglichen, die in bilingualen Wörterbüchern als äquivalent angegeben werden. Mit der angewandten Methode lassen sich fehlerhafte Äquivalenzangaben ermitteln und korrigieren.

Schlüsselwörter: Rechtsterminologie, Begriffsdistribution, Äquivalenz von juristischen Termini, juristische Rechtswörterbücher

\section{The juristic analysis of terminological equivalence in bilingual dictionaries of legal terms}

The article is devoted to the issue of terminological equivalents that can be found in bilingual dictionaries. The aim of the analysis is to verify the accuracy of equivalents provided by a dictionary from a legal perspective and to formulate methodological implications that may be useful for lexicographers and translators when searching for the accurate equivalents of a legal character.

Keywords: legal terminology, distribution and conceptualizations of legal terms, methodology of contrastive studies

Łukasz Iluk

Wyższa Szkoła Finansów i Prawa w Bielsku-Białej

ul. Tańskiego 5

43-382 Bielsko-Biała

Polen

E-Mail: lukasz@iluk.katowice.pl 